\title{
A Study of Image Segmentation Based on Level Set Method ${ }^{*}$
}

\author{
Xiaowei Chang, Daowang Li, Yihong Xu \\ School of information engineering, Shandong Management University, No. 60, Jinan, China \\ ise_changxw@126.com, lidaowang6045@163.com,xuyhong2002@163.com
}

\begin{abstract}
Geometric active contour model has many advantages over parametric active contour model, such as computational simplicity and the ability to change curve topology during deformation, et al. The level set method is a class of curve evolution methods based on the geometry active contour model. Level set method coupled with curve evolution theory conquers many limitations of traditional snakes, which widens the utilization of active contours model. Moreover, by analyzing latest level set method, this paper summarizes the research, development and applications of active contour model. Finally, this paper points out future research orientations on the theories and applications research of level set method. mode.

Index Terms - Image segmentation, level set, active contour
\end{abstract}

\section{Introduction}

The main goal of image segmentation is to divide an image into parts that have a strong correlation with objects or areas of the real world contained in the image [1]. Broadly speaking, there are two main groups of deformable contour/surface models: parametric deformable models and geometric deformable models [2-5]. The deformation difference of parametric deformable models and geometric deformable models is that the former represent curves and surfaces explicitly in their parametric forms, but the latter represent curves and surfaces implicitly.

Parametric deformable models have gained significant attention and development since its first introduction by Kass, Witkin, and Terzopoulus, and have been used in a range of applications, including edge detection, object recognition, shape modeling, and motion tracking [2,6,7]. Examples include the traditional deformable model, the balloondeformable model, and the gradient vector flow (GVF) model et al [8].

Geometric deformable models, based on curve evolution theory and the level set method [2, 9], were proposed independently by Caselles et al. [3] and Malladi et al. [4] and named level set front propagation and geodesic active contour segmentation approaches. The level set technique is a method to represent shapes and track moving interfaces. The ability of handling local deformations, multicomponent structures, and changes of topology are its major strengths $[1,9]$.

\section{Level Set Method}

The basic idea of level set methods is to change the movement track of a planar curve into the movement track of a three-dimensional surface. Its advantages are as follows: parameter-free representation, topological flexibility, and the ability to handle local deformations.

\section{A. Curve evolution theory}

The curve evolution theory studies the deformation of curves using geometric measures such as the unit normal and curvature. Let us consider a moving curve $X(s, t)=[X(s, t), Y(s, t)]$, where $\mathrm{s}$ is any parameterization and $\mathrm{t}$ is the time, and denote its inward unit normal as $\mathrm{N}$ and its curvature as $\kappa$, respectively. Let the curve develop along its normal direction according to the partial differential equation

$$
\frac{\partial X}{\partial t}=V(\kappa) \mathbf{N}
$$

Where $V(\kappa)$ is called speed function, since it determines the speed of the curve evolution.

The curvature deformation and constant deformation are two mainly studied curve deformation in curve evolution theory

\section{B. Level set method}

The level set method evolves a curve by updating the level set function at fixed coordinates through time. A useful property of this approach is that the level set function remains a valid function while the embedded curve can change its topology.

Given a level set function $\phi(\mathrm{x}, \mathrm{y}, \mathrm{t})$ with the contour $\mathrm{X}(\mathrm{s}, \mathrm{t})$ as its zero level set, the situation is described by

$$
\phi(\mathrm{X}(\mathrm{s}, \mathrm{t}), \mathrm{t})=0 .
$$

If this equation is differentiated with respect to $t$ and the chain rule is used,

$$
\frac{\partial \phi}{\partial t}+\nabla \phi \cdot \frac{\partial \mathrm{X}}{\partial t}=0
$$

We assume that $\phi$ is negative inside the zero level set and positive outside. The inward unit normal to the level set curve is given by

$$
\mathbf{N}=-\frac{\nabla \phi}{|\nabla \phi|}
$$

\footnotetext{
* This work was supported by the Spark Plan from Science and Technology Department of Shandong Province under Grant No.2012XH17002 and the Construction Plan of E-government from the Economic and Information Technology Commission of Shandong Province under Grant No. 2150511.3168.
} 
Using this fact and (1), we can rewrite (2) as

$$
\frac{\partial \phi}{\partial t}=\nabla \phi \cdot \frac{V(\kappa) \nabla \phi}{|\nabla \phi|}
$$

Where the curvature kat the zero level set is given by

$$
\kappa=\nabla \cdot \frac{\nabla \phi}{|\nabla \phi|}=\frac{\phi_{x x} \phi_{y}^{2}-2 \phi_{x} \phi_{y} \phi_{x y}+\phi_{y y} \phi_{x}^{2}}{\left(\phi_{x}^{2}+\phi_{y}^{2}\right)^{3 / 2}}
$$

Equation (5) shows how to perform curve evolution specified by (1) using the level set method.

\section{Parametric deformable models}

\section{A. Traditional Deformable Model}

The active contour model, or snake, was proposed by Kass, Witkin, and Terzopoulos in 1987. Snake is the classical parametric deformable models and may be understood as a special case of a more general technique of matching a deformable model to an image by means of energy minimization. A snake is an energy-minimizing spline guided by external constraint forces and influenced by image forces that pull it toward features such as lines and edges. Snakes are active contour models: they lock onto nearby edges, localizing them accurately [2]. The main purpose of the energy minimizing formulation of deformable contours is to find a parameterized curve. The parameterized curve minimizes the weighted sum of internal energy and potential energy.

The traditional deformable model [2] is defined as $\mathrm{v}(\mathrm{s})=$ $(\mathrm{x}(\mathrm{s}), \mathrm{y}(\mathrm{s}))$, where $\mathrm{x}(\mathrm{s})$ and $\mathrm{y}(\mathrm{s})$ are $\mathrm{x}$ and $\mathrm{y}$ coordinates along the contour and $s \in[0,1]$. The minimized energy function may be written as

$$
E_{\text {snake }}^{*}=\int_{0}^{1}\left(E_{\text {int }}(\mathbf{v}(s))+E_{\text {image }}(\mathbf{v}(s))+E_{\text {con }}(\mathbf{v}(s))\right) d s
$$

where $E_{\text {int }}$ represents the internal energy of the spline, $E_{\text {image }}$ represents image forces, and $E_{c o n}$ external constraint forces.

The internal spline energy can be written

$$
E_{\text {int }}=\frac{\left(\alpha(s)\left|\mathbf{v}_{s}(s)\right|^{2}+\left(\beta(s)\left|\mathbf{v}_{s s}(s)\right|^{2}\right)\right.}{2} .
$$

A snake that minimizes E must satisfy the Euler equation [10]

$$
\alpha \mathbf{V}^{\prime \prime}(s)-\beta \mathbf{V}^{\prime \prime \prime}(s)-\nabla E_{\text {ext }}=0 .
$$

The (7) can be minimized as

$$
E_{\text {snake }}^{*}=\int_{0}^{1} E_{\text {snake }}(\mathbf{v}(s)) d s .
$$

The Euler-Lagrange condition states that the spline v(s) must satisfy

$$
\frac{d}{d s} E_{\mathbf{V}_{s}}-E_{\mathbf{v}}=0
$$

where $E_{\mathbf{V}_{s}}$ is the partial derivative of $E$ with respect to $\mathrm{dv} / \mathrm{ds}$ and $E_{\mathbf{v}}$ is the partial derivative of $E$ with respect to $\mathrm{v}$ [11]. Then, the partial derivative of $\mathbf{V}$ with respect to $t$ is then set equal to the left hand side of (9) as follows:

$$
\mathbf{V}_{t}(s, t)=\alpha \mathbf{V}^{\prime \prime}(s)-\beta \mathbf{V}^{\prime \prime "}(s)-\nabla E_{e x t} .
$$

Internal forces and potential forces are obtained by minimizing the total energy. Internal forces hold the curve together and external forces attract the curve toward the desired object boundaries. Parametric curves are initialized within the image domain and forced to move toward the potential energy minima to find the object boundary.

There are many problems about minimization of the snake energy integral, such as designing numerous parameters, providing reasonable initialization, the solution's numerical instability of the Euler-Lagrange equation. Several variants of active contour models emerged to improve the original method's performance. The main improvements include finite element snakes, B-snakes, Fourier deformable models, united snakes, and GVF snakes, etc.

\section{B. Balloon Deformable Model}

Balloon Deformable Model resolves the difficulties with the small capture region of the traditional deformable model and overcomes isolated energy valleys resulting from spurious edge points. Cohen and Cohen [12] define

$$
E_{e x t}=-\nabla P(\mathrm{x})
$$

where $\mathrm{P}$ is the potential function calculated using a Euclidean distance map. This approach is more effective in the case of closed or nearly closed contours, but it still fails to segment nuclei accurately.

\section{GVF Deformable Model}

Gradient vector flow (GVF) fields and their use in snake image segmentation were reported in [8]. The GVF is used as an external force in the snake (7) forming a GVF snake. The GVF deformable model provides large capture regions and the GVF vector flow enables the deformable model to enter into convex regions within the image.

The GVF field $g(x, y)=(u(x, y), v(x, y))$ minimizes the energy functional

$$
E=\iint \mu\left(u_{x}^{2}+u_{y}^{2}+v_{x}^{2}+v_{y}^{2}\right)+|\nabla f|^{2}|g-\nabla f|^{2} d x d y .
$$

The parameter $\mu$ is a regularization parameter governing the tradeoff between the first term and the second term in the integrand. The GVF field can be found by solving the following Euler equations

$$
\begin{aligned}
& \mu \nabla^{2} u-\left(u-f_{x}\right)\left(f_{x}^{2}+f_{y}^{2}\right)=0 \\
& \mu \nabla^{2} v-\left(v-f_{y}\right)\left(f_{x}^{2}+f_{y}^{2}\right)=0
\end{aligned}
$$


where $\nabla^{2}$ is the Laplacian operator. (15a) and (15b) can be solved by treating and as functions of time and solving the following two decoupled equations for $t \rightarrow \infty$ :

$$
\begin{aligned}
& u_{t}(x, y, t)=\mu \nabla^{2} u(x, y, t) \\
& -\left(u(x, y, t)-f_{x}(x, y)\right)\left(f_{x}(x, y, t)^{2}+f_{y}(x, y, t)^{2}\right) \\
& v_{t}(x, y, t)=\mu \nabla^{2} v(x, y, t) \\
& -\left(v(x, y, t)-f_{x}(x, y)\right)\left(f_{x}(x, y, t)^{2}+f_{y}(x, y, t)^{2}\right)
\end{aligned}
$$

Fig. 1 gives an example of a CT (computed tomography) image segmentation using GVF snakes.

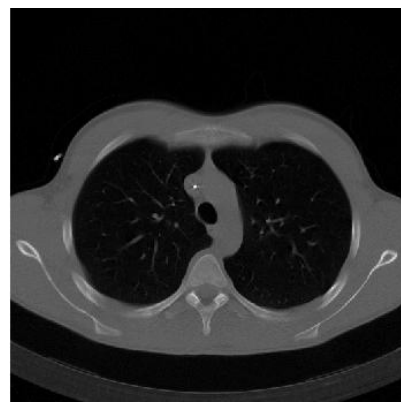

(a)

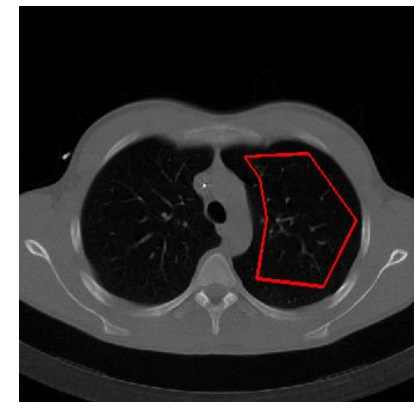

(b)

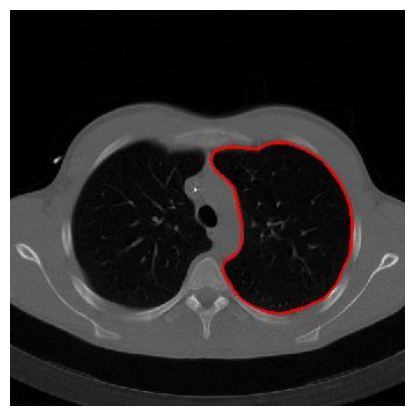

(c)

Fig. 1 Lung segmentation using GVF snakes.
(a) Original lung image
(b) Initialization to GVF snakes.
(c) Result obtained using GVF snakes

\section{Geometric deformable models}

\section{A. Models based on gradient}

Caselles et al., Kichenassamy et al., and Paragios developed another classical snake model, the geodesic active contour, as the geometric alternative to the original snake [5, 13, 14]. This geodesic approach allows to connect classical "snakes" and geometric active contours, and moreover the approach has the following main properties:

1) Improves existing curve evolution models.

2) Allows simultaneous detection of interior and exterior boundaries in several objects.

3) Holds formal existence, uniqueness, stability, and consistency results.

4) Does not require special stopping conditions [15].

\section{B. Models based on Mumford-Shah functional}

Chan and Vese proposed a model, based on techniques of curve evolution, Mumford-Shah functional for segmentation, and level sets. Chan-Vese model can detect objects whose boundaries are not necessarily defined by gradient. The ChanVese model can be classified into two subsidiary sets: piecewise smooth Chan-Vese model and piecewise constant Chan-Vese model. The advantages of the model are as follows: the ability of detecting smooth boundaries, scale adaptivity, automatic change of topology, and robustness with respect to noise.

Considering a 2D image consisting of pixels $\mathrm{I}(\mathrm{x}, \mathrm{y})$ and the segmentation defined by an evolving closed zero-level set curve $\phi$, the Chan-Vese energy functional is shown in Eq. (7).

$$
\begin{aligned}
C(\phi, \alpha 1, \alpha 2)= & C_{1}\left(\phi, \alpha_{1}, \alpha_{2}\right)+C_{2}\left(\phi, \alpha_{1}, \alpha_{2}\right) \\
= & \int_{\text {inside } \phi)}(I(x, y)-\alpha 1)^{2} d x d y \\
& +\int_{\text {outsid }(\phi)}(I(x, y)-\alpha 2)^{2} d x d y
\end{aligned}
$$

Where $\alpha_{1}, \alpha_{2}$ represent the mean intensities of the interior and exterior of the segmented object(s). The relation between the curve and the object is shown as follows.

1) The curve $\phi$ is outside the object. In this case $C_{1}(\phi)>0$ and $C_{2}(\phi) \approx 0$.

2) The curve $\phi$ is inside the object. In this case $C_{1}(\phi) \approx 0$ and $C_{2}(\phi)>0$.

3) The curve $\phi$ is both inside and outside the object. In this case $C_{1}(\phi)>0$ and $C_{2}(\phi)>0$.

4) The curve $\phi$ is coincides with the object boundary. In this case $C_{1}(\phi) \approx 0$ and $C_{2}(\phi) \approx 0$.

Fig. 2 gives an example of an X-ray image of the hand segmentation using Chan-Vese model.

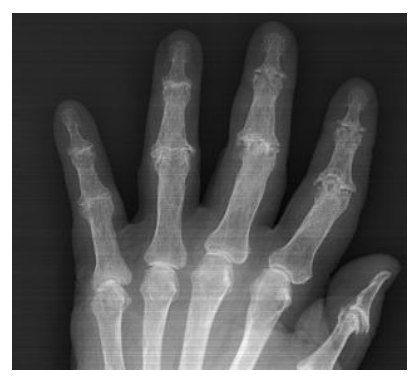

(a)

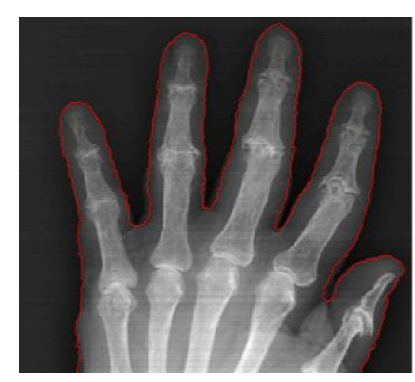

(b)
Fig. 2 Hand segmentation using Chan-Vese model. (a) Original hand image

(b) Result obtained using Chan-Vese model.

Promising approaches have been proposed recently[1617], such as the work by Shang Yan-feng, WANG Ning, and WANG Hui [18], who developed a hybrid method based on regional energy minimization and active contour model to allow more effective control of the topology. 


\section{Conclusion}

In this paper, we have described the fundamental formulation of image segmentation based on level set method. Level set image segmentation method based on gradient applies to the image with great gradient diversity, while method based on Mumford-Shah functional applies to the image with noise. In future work, further improvements in deformable models will be made in model representation, model training and learning, and model performance validation. The main goal of this paper is to help researcher to understand the level set based segmentation method. This paper will be helpful to those who want to apply level set method into their research.

\section{VI . Acknowledgment}

We thank Mr. Tomáš Svoboda, Mr. Jan Kybic, and Mr.Václav Hlaváč for the use of the original images used in this paper.

\section{References}

[1] Osher S, Sethian J A, "Fronts propagating with curvature dependent speed: algorithms based on Hamilton-Jacobi formulations," Journal of Computational Physics, Vol. 79, no.1, pp. 12-49, 1988.

[2] M. Kass, A. Witkin, and D. Terzopoulos, "Snakes: active contour models, "Int'l J. Comp. Vis., vol.1, no. 4, pp. 321-331, 1988.

[3] V. Caselles, F. Catte, T. Coll, and F. Dibos, "A geometric model for active contours in image processing," Numerische Mathematik, Vol.66, no.1, pp.1-31, 1993.

[4] R. Malladi, J. A. Sethian, and B. C. Vemuri, "Shape modeling with front propagation: a level set approach," IEEE Trans. Patt. Anal. Mach. Intell., Vol. 17, no.2, pp.158-175, 1995.

[5] V. Caselles, R. Kimmel, and G. Sapiro, "Geodesic active contours," in Proc. 5th Int'l Conf. Comp. Vis., pp. 694-699, 1995.
[6] Leymarie F, Levine MD, "Tracking deformable objects in the plane using an active contour model," IEEE Trans Pattern Anal Machine Intell, Vol.15, pp.617-634, 1993.

[7] Terzopoulos D, "The computation of visible-surface representations," IEEE Trans Pattern Anal Machine Intell , vol. 10, no. 4, pp.417-438, 1988.

[8] Xu C, Prince JL, "Snakes, shapes, and gradient vector flow," IEEE Trans Image Process, Vol. 7, no.3, pp.359-369, 1998.

[9] J. A. Sethian, Level Set Methods and Fast Marching Methods: Evolving Interfaces in Computational Geometry, Fluid Mechanics, Computer Vision, and Material Science, Cambridge University Press, 2nd ed., Cambridge, UK, 1999, References 171.

[10] Xu, C., \& Prince, J. L. , "Snakes, shapes, and gradient vector flow," Image Processing, IEEE Transactions on, vol.7, no.3, pp.359-369,1998

[11] Sonka, M., Hlavac, V., \& Boyle, R., Image processing, analysis, and machine vision. Thomson-Engineering, 2007.

[12] Cohen, L. D., "On active contour models and balloons," CVGIP: Image understanding, vol.53, no.2, pp.211-218, 1991.

[13] Kichenassamy S, Kumar A, Olver PJ, Tannenbaum A, Yezzi AJ, "Gradient flows and geometric active contour models," Proc. 5th Int'l Conf. Comp. Vis., pp. 810-815, 1995.

[14] Paragios N, Deriche R, "Geodesic active contours and level sets for the detection and tracking of moving objects," IEEE Trans Pattern Anal Machine Intell, vol.22, no.3, pp.266-280, 2000.

[15] Caselles V, Kimmel R, Sapiro G,"Geodesic active contours," International Journal of Computer Vision, vol. 22, no.1, pp.61 79, 1997.

[16] Estellers, V., Zosso, D., Rongjie Lai, Osher, S., Thiran, J., Bresson, X, "Efficient Algorithm for Level Set Method Preserving Distance Function, "Image Processing, IEEE Transactions on, vol.21, no. 12, pp.4722-4734, 2012.

[17] YANG Chang-jun, YANG Xin, "Abdominal CT Image Segmentation Based on Graph Cuts and Fast Level Set," CT Theory and Applications, vol.20, no. 3, pp.291-300, 2011.

[18] SHANG Yan-feng, WANG Ning, WANG Hui, "Medical object extraction model based on regional energy minimization and active contour model," Application Research of Computers. Vol.29, no.7, pp.2715-2718, 2012. 